\title{
INVESTIGATION OF THE MICROTEK SCANMAKER 1000XL PLUS SCANNER OF THE INSTITUTE OF ASTROPHYSICS OF THE ACADEMY OF SCIENCES OF THE REPUBLIC OF TAJIKISTAN
}

\author{
A.Mullo-Abdolov ${ }^{1}$, G.Kokhirova ${ }^{1}$, H.Relke ${ }^{2}$, Q.Yuldoshev ${ }^{3}$, Yu.Protsyuk ${ }^{4}$, V.Andruk ${ }^{5}$ \\ ${ }^{1}$ Institute of Astrophysics of AS of Republic Tajikistan,aziz.sherzod@gmail.com \\ ${ }^{2}$ Walter Hohmann Observatory, 159, 45133 Essen, Germany, helena_relke@yahoo.com \\ ${ }^{3}$ Ulugh Beg Astronomical Institute of the Uzbek Academy of Sciences, 33 Astronomicheskaya \\ St., 100052 Tashkent, Uzbekistan,qudratillo@astrin.uz \\ ${ }^{4}$ Research Institute "Mykolaiv Astronomical Observatory”, 1 Observatornaya St., 54030, \\ Mykolaiv, Ukraine,yuri@nao.nikolaev.ua \\ ${ }^{5}$ Main Astronomical Observatory of the National Academy of Sciences of Ukraine, 27 \\ Akademika Zabolotnoho St., 03680 Kyiv, Ukraine,andruk@mao.kiev.ua
}

\begin{abstract}
This work was done to estimate the accuracy of the processing method of photographic plates and the detailed study of the Microtek ScanMaker 1000XL Plus scanner, which plans to be used for the digitizing of about 1600 photographic plates of the Astrophysics Institute of the Academy of Sciences of the Republic of Tajikistan (Dushanbe) recorded for the FON project. For the processing of these photographic plates will be used software created in the LINUX/MIDAS/ROMAFOT environment. Six sequential scans of a single plate (1150, the Pleiades cluster) with a spatial resolution of $1200 \mathrm{dpi}$ were processed to evaluate the repeatability of the scanner's astrometric and photometric errors. The size of photographic plates is $8 \times 8$ degrees $(30 \times 30 \mathrm{~cm}$ or $13000 \times 13000$ pixels). The estimated errors of the scanner for the stars brighter than $\mathrm{B}<13.5^{\mathrm{m}}$ are $\sigma_{\mathrm{xy}}= \pm 0.054$ pixels for the rectangular coordinates and $\sigma_{\mathrm{m}}=$ $\pm 0.020^{\mathrm{m}}$ for the instrumental magnitudes. The estimated astrometric accuracy of the processing of stellar fields of photographic plates in the system of the Tycho- 2 catalogue is $\sigma \alpha \delta= \pm 0.13^{\prime \prime}$.
\end{abstract}

Keywords: photographic plates, scanning, processing of digitized plates, astrometry, photometry, data analysis.

\section{The Photographic Survey of the Northern Sky - FON project}

The plan of the photographic observation of the Northern Sky (FON project) was proposed in 1976 by Main Astronomical Observatory of Ukraine (Goloseevo) scientists I.H.Kolchinskii and A.B.Onegina (Kolchinskii, 1977). The detailed overview of the current stage of the FON project was done by L.K.Pakuljak (Pakuljak, 2016). The idea of the FON project emerged from the state of photographic astrometry in the early 1970s. Suffice to say that the coordinate systems which were built in the past were significantly worsened because of the inaccuracies of the proper motions. It was necessary to create a homogeneous coordinate system that would be able to solve the problems of the modern astrometry, astronomy and cosmology. The main factors contributing to the development of the project were:

1. The completion of the Carte du Ciel project $(\mathrm{CdC}$, Map of the Sky), started at the end of the 19th century and resulted with the creation of an accuracy stars catalog down to the 11th magnitude and a photographic stars atlas down to the 14th magnitude. As the result of the digitization of $\mathrm{CdC}$ data performed in the 1980s at the Sternberg Astronomical Institute (GAISh) was created an Astrographic Catalogue (AC) of 4 millions stars on the epoch 1950.0. The idea of the re-observing the sky with modern instruments was very attractive. Moreover the difference of more than 70 years between the observing epochs gave the opportunity to became proper motions for a large number of stars with the greatest accuracy at that time.

2. The development of an algorithm to handle an array of overlapping fields as a whole, using variables that describe the general as well as the individual parameters of the photographic plates (Google, Eichhorn \& Lucas, 1970). As was shown by Eichhorn the solution of the system with tens thousands of unknowns taken into account the instrumental aberrations ensured on the best way the uniformly accuracy of the catalogue created on the base of overlapping fields.

3. Almost simultaneously in the 1970 s some astronomical observatories of the former Soviet Union were equipped with the identical wide-angle Astrographs produced by the Carl Zeiss Company (Jena, Germany) with an aperture of $40 \mathrm{~cm}$ and focal distances of 2 or 3 meters. The observatories taking part in FON project: Holosievo, Zelentschuk, Zvenigorod, Dushanbe, Abastumani and Kitab were divided into four groups. The North Sky must be photographed four times with an overlapping. Each group photographed the sky independently according to a common schema with a shift of the centres of the fields on 2 degrees in such a way to get the fourfold overlapping. Observations were made with an exposure to became suitable for the measurement images of 
stars down to 16-17 magnitude. Additional short exposure with a shift of the centre of the field on both coordinates was made on the same plate to correct the brightness equation. Such work distribution between the observatories gave advantages to the FON project compared with the zone observations. Each star would be photographed at least four times by different telescopes, which is very important to avoid systematic errors in the future catalogue.

\section{History of the realization of the FON project on the GisAO, Dushanbe, Tadjikistan}

The Gissar Observatory of the Institute of Astrophysics of the Academy of Sciences of the Republic of Tajikistan (GissAO) also participated in the implementation of the FON project. Regular observations began in 1985 and continued until 1992. The observations were carried out by Hamburg O., Mullo-Abdolov A., Relke E., Tsyhankova M., Kiselev N. The 1578 photographic plates were recorded. During this period by Relke H. was prepared a dissertation, which was successfully defended in April 1993 at the Goloseevo Observatory, Kyiv. In the frame of the thesis "Creating an astrometric standard in the Swan constellation" were developed and applied the algorithms for the reduction of the photographic plates of the FON project. The scientific leader of the dissertation was Dr. Kislyuk Vitaly Stepanovich, the head of the astrometry department of the Main Astronomical Observatory of Ukraine (Goloseevo). The main results were published in the following works (Kislyuk, Relke, 1992; Relke, Gamburg, 1993; Relke, 1993; Relke, 1991).

Owing to the unstable political situation of the 1990s, the lack of financial resources and the forced departure of almost 75 percent of the scientific staff, all scientific projects of the Institute of Astrophysics were interrupted including the observation for the FON project. During this time the observatories of the Institute of Astrophysics were plundered, almost all costly telescopes and equipment were severely damaged.

After the end of the Civil war the remaining staff of the Institute of Astrophysics with the help and support of russian specialists took over the rehabilitation of the institute's observatories. The telescopes of the GissAO and Sanglok observatory were repaired and equipped with modern technologies. Thanks to the staff of the Institute of Astrophysics all photographic plates of the FON project were retained and moved to a safe place, where they have been stored for more than 20 years. All photographic plates are in good condition. Over the years of the existent of the Institute of Astrophysics a huge photographic material was been accumulated. The modern technologies allow now the processing of all accumulated material on a new more higher level. Thanks to the support of the Academy of Sciences of Tadjikistan the Institute of Astrophysics was able to acquire a modern scanner, which plans to be applied for the scanning of the institute's photographic material to create a digital library. On the basis of the scanned photographic plates of the FON project is planned the creation of a catalogue of the Gissar Observatory. The scientists of the observatories of Goloseevo and Nikolayev (Ukraine), Kitab (Uzbekistan) and Walter Hohmann (Germany) have provided significant support in the instructing of using of scanner and processing of photographic plates for the FON project. So, at the Ulugh Beg Astronomical Institute of the Uzbek Academy of Sciences Mullo-Abdolov was trained in the using of the scanner and it's basis software. The scientists of the Goloseevo observatory have provided the software developed for the processing of scanned images. Digitized images are planned to be processed at Goloseevo, Nikolayev, Kitab, Walter Hohmann and GissAO observatories. The first step in the digitization and processing of photographic plates is the study of the astrometric and photometric characteristics of the scanner.

\section{Errors of the Microtek ScanMaker 1000XL Plus scanner}

In this work for the investigation of the astrometric and photometric characteristics of the Microtek ScanMaker 1000XL Plus scanner the authors used the procedures previously applied in the series of works (Golovnya, 2010; Eglitis, 2017; Muminov, 2013; Protsyuk, 2014b). The developed software for the processing of the digitized images is described in publications (Andruk, 2015a; Andruk, 2017; Protsyuk, 2014a). Six consecutive scans of the same plate number 1150 photographed with two exposures (1 and 27 minutes) were processed to estimate the astrometric and photometric errors of the scanner (Andruk, 2012).
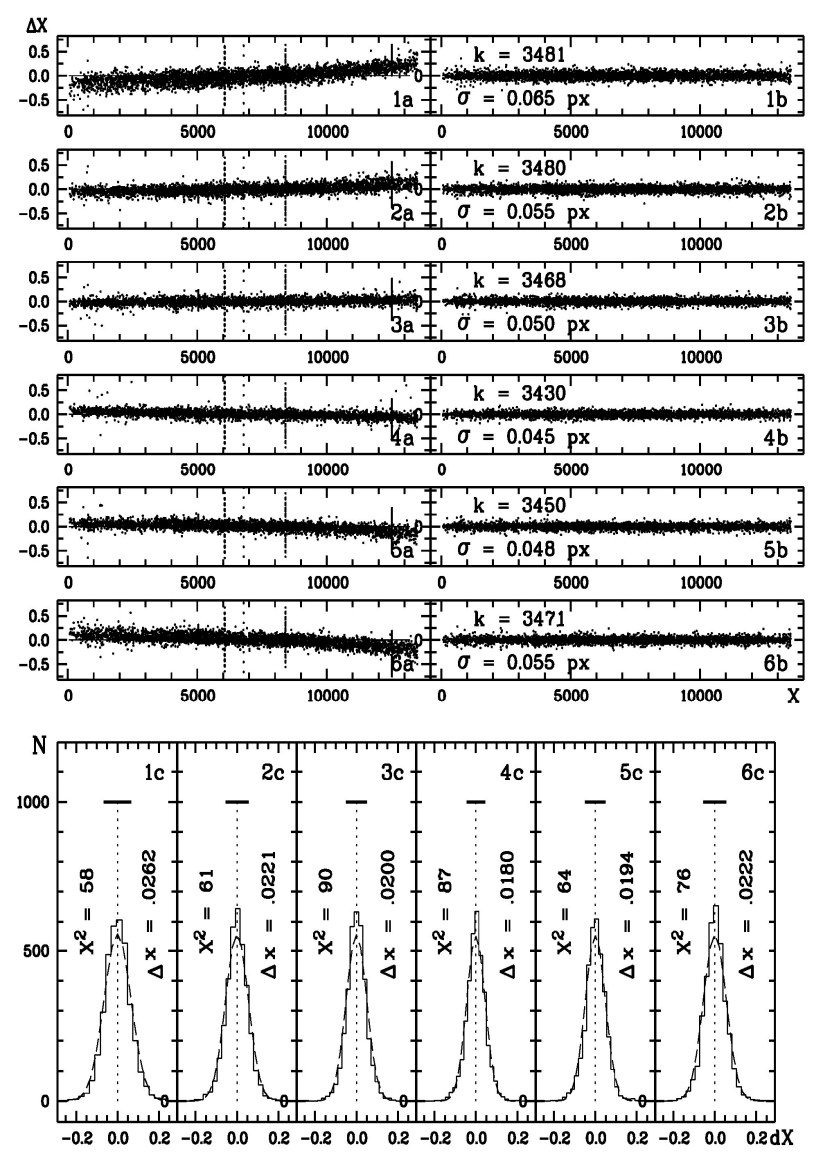

Figure 1: The trends of the differences between the sixconsecutive scans and the imaginary average scan for the X-coordinates. 
The scanning was completed with a spatial resolution of 1200 dpi (Protsyuk, 2014c). The performing of calculations of all registered bright objects (approximately 3450) was done in the following way: the average values for the rectangular coordinates $\mathrm{X}, \mathrm{Y}$ as well as for the instrumental magnitudes $\mathrm{m}$ of all objects were obtained by averaging of the corresponding valuesfor all objects over the six scans. On such a way we created an imaginary average scan. On the left side of Figure 1 are illustrated the trends of differences between the six consecutive scans and the imaginary average scan calculated for the X-coordinates (1a, 2a, 3a, 4a, 5a, 6a). After the correction of differences of each scan for the systematic errors we have results are shown on the right side of the Figure 1 (1b, 2b, 3b, 4b, 5b, $6 b)$. The values of the standard deviation (SD) of one difference of coordinate for all six scans are indicated on the Y-axis. At the bottom of the Figure 1 are shown the frequency distributions of these differences. Differences are given in the form of real (continuous lines) and theoretical (dashed lines) distribution functions on appropriate intervals. The values of the length of intervals $\Delta x$ and the values

$\mathrm{X}^{2}$ are shown on the right and on the left respectively. The corresponding trends of the differences for the Y-coordinates are given in the Figure 2 and for the instrumental magnitudes $\mathrm{m}$ in the Figure 3.
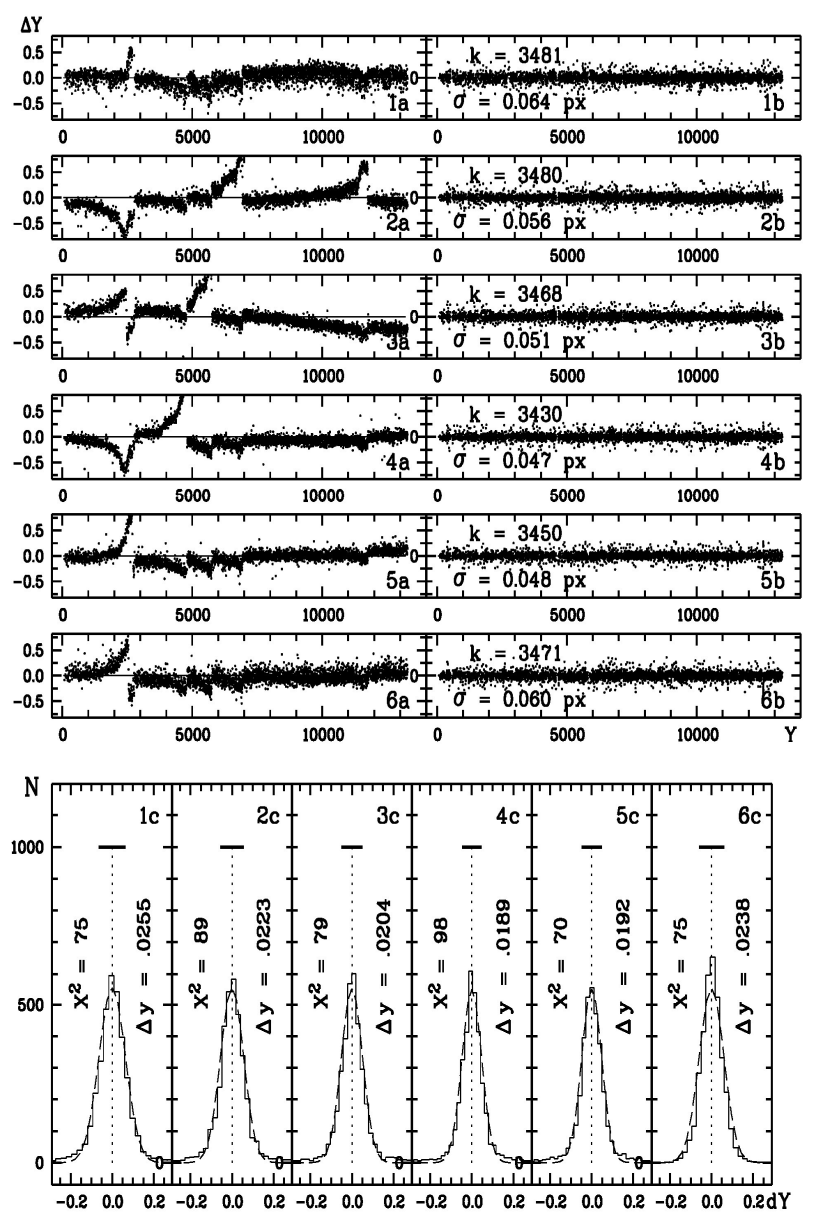

Figure 2: The trends of the differences between the six consecutive scans and the imaginary average scan for the Y-coordinates.
The trends of the astrometric differences for the Ycoordinate have any breaks, which is probably due to the irregular movement of the carriage with the CCD linear. The results are shown in Figure 1, 2 and 3 were obtained for the stars having the B magnitudes brighter than $13.5^{\mathrm{m}}$. As you can see the errors of one definition of differences for the rectangular coordinates are $\sigma_{x y}=0.045$ pixels and 0.066 pixels, errors in the definition of magnitudes do not exceed the values of $\sigma_{\mathrm{m}}=0.018^{\mathrm{m}}$ and $0.022^{\mathrm{m}}$.

By transform to the equatorial coordinate system it is necessary to take into account the scale factor of 2.2. By transform to the Johnson system, the instrumental magnitudes should be corrected for the scale factor of 1.5 due to the contrast of the photo emulsion. Taking into account the scale factors the following conclusion can be made: The using of the flatbed Microtek ScanMaker 1000XL Plus scanner together with the developed software enables the digitizing and processing of the photographic plates as well as the obtaining of characteristics of the objects (high, moderate and faint brightness) with internal errors better than 0.1 " and $0.04^{\mathrm{m}}$. For the more finer objects $\left(\mathrm{B}=16^{\mathrm{m}}-17.5^{\mathrm{m}}\right)$ these errors could be approximately twice worser. This result is based on the earlier studies for the photographic plates of the $30 \times 30 \mathrm{~cm}$ size with the 1200 dpi scanning modes.
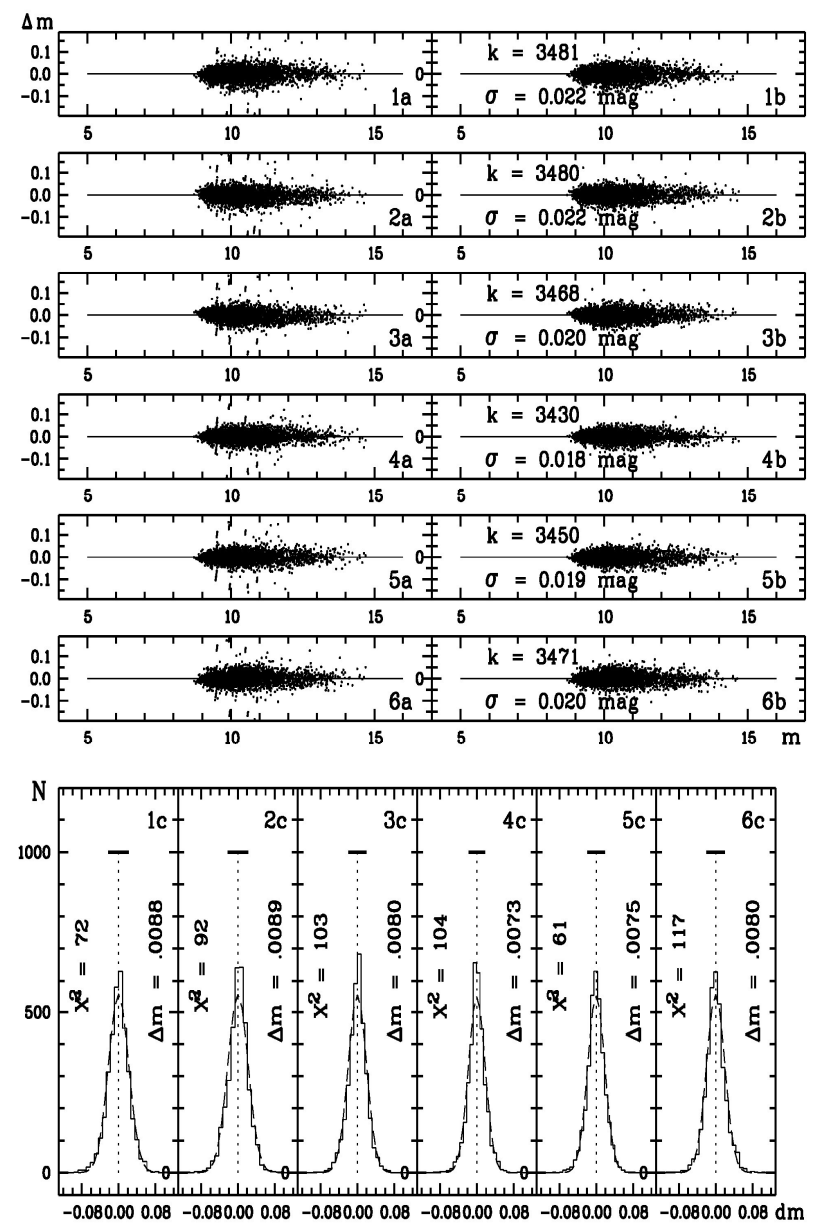

Figure 3: The trends of the differences between the six consecutive scans and the imaginary average scan for the instrumental magnitudes $\mathrm{m}$. 
The six top panels on the left of the Figure 4 show the trends of the scanner's systematic errors $(1 \mathrm{a}, 1 \mathrm{~b}, 1 \mathrm{~d}, 1 \mathrm{e})$ for the coordinates and the brightness equation $(1 \mathrm{c}, 1 \mathrm{f})$ before the correction for the scanner's systematic errors. On the right the corresponding reduction errors after the correction for the scanner's systematic errors, brightness equation as well as for the aberrations of the telescope optics. Two lower panels illustrate the distribution of the reference stars from the Tycho-2 catalogue over the field of the plate No. 1150 (left) and the characteristic curve in the system of B-magnitudes Tycho- 2 catalogue.
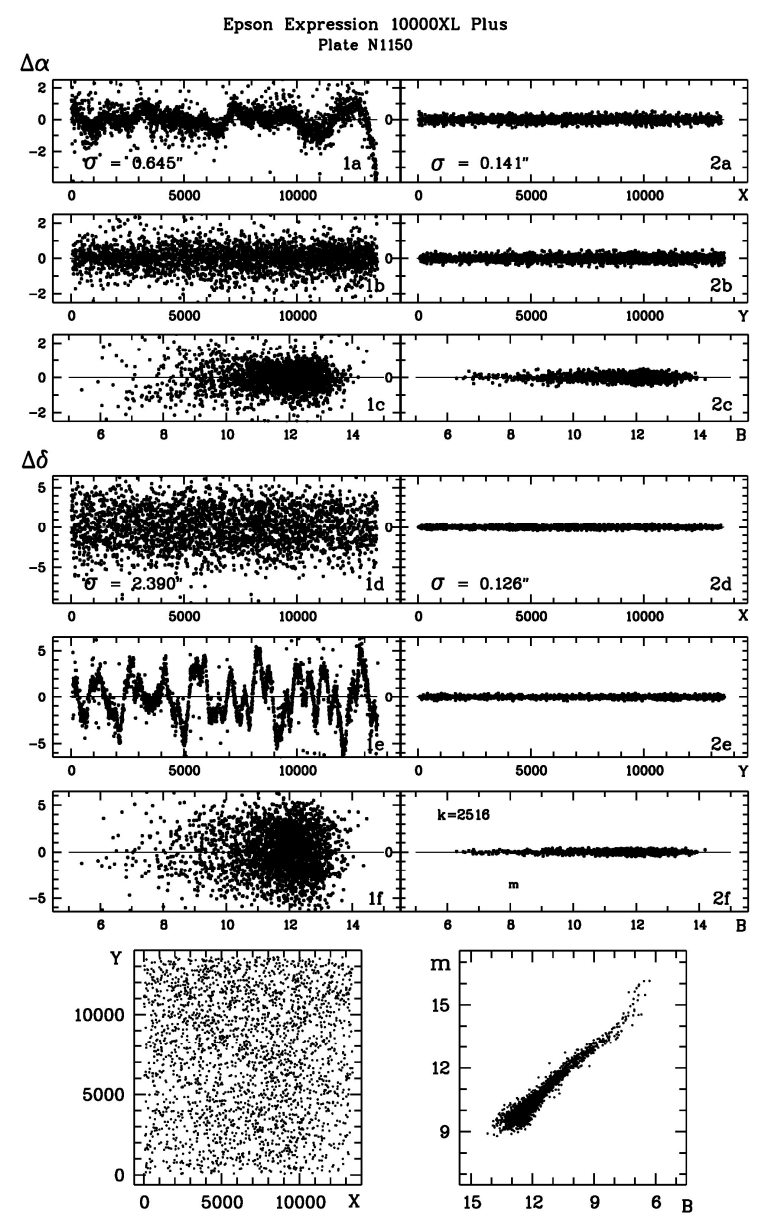

Figure 4: On the left: the trends of the systematic errors for coordinates $(1 \mathrm{a}, 1 \mathrm{~b}, 1 \mathrm{~d}, 1 \mathrm{e})$ and the brightness equation (1 c, 1f) before the correction for the scanner's systematic errors. On the right: the corresponding reduction errors after the correction for the scanners systematic errors, brightness equation as well as for the aberration of the telescope optics. Two lower panels show the distribution of the reference stars from the Tycho- 2 catalogue over the field of the plate No. 1150 (left) and the characteristic curve in the system of B-magnitudes Tycho-2 catalogue.

\section{Conclusion}

The results of the performed work concerning the astrometric and photometric characteristics of the processing of photographic plates digitized by the Microtek ScanMaker 1000XL Plus scanner allowed to make the following conclusion: Within the precision of reduction of photographic plates the scanner is suitable for the performing of astrometric and photometric works for the terrestrial astronomy and in particular for the FON project (Andruk, 2014; Andruk, 2015b; Andruk, 2016a; Andruk 2016b; Pakuliak, 2016; Relke, 2015; Yuldoshev, 2017). Errors of one measurement are 0.1 arc seconds for the equatorial coordinates and $0.04^{\mathrm{m}}$ for the B-magnitudes.

Acknowledgements. The authors are thankful to academician Farhod Rahimi, the President of Academyof Sciences of the Republic of Tajikistan, for the supporting of the project "Creating of the digital archive of the GissAO" and help by the acquirement of the scanner. The photographic plates which were recorded for the FON project could be scanned and on the base of these digitized images will be created a new catalogue of the north sky down to the 17 th magnitude.

\section{References}

Andruk V.M., Ivanov G.A., Yatsenko A.I. et al.: 2012, Bull. T. Shevchenko Nat. Univ. Kyiv., Astron. N48, 11 (in Ukraine)

Andruk V.N., Golovnya V.V., Ivanov G.A. et al.; 2014, Odessa Astron. Publ., 27, 53.

Andruk V.N., Pakuliak L.K., Golovnya V.V. et al.: 2015, preprint, arxiv.org/abs/1512.05535.

Andruk V.M., Pakuliak L.K., Golovnia V.V. et al.; 2015, Odessa Astron. Publ., 28, 192.

Andruk V.M., Golovnia V.V., Ivanov G.A. et al.: 2016, Kinem. Phys. Cel. Bodies, 32, N1, 38.

Andruk V.M., Pakuliak L.K., Golovnia V.V. et al.: 2016, Kinem. Phys. Cel. Bodies, 32, N5, 260.

Andruk V.M., Pakuliak L.K., Golovnia V.V. et al.: 2017, Science and Innovation, 13a, N1, 17.

Eglitis I., Andruk V.: 2017, Open Astronomy, 26, N1, 7.

Golovnya V., Andruk V., Yatsenko A.: 2010, J of Phys. Studies, 14, N2, 2902 (in Ukraine).

Googe, W. D., Eichhorn, H., Lucas, C. F. Mon.Not.R. Astr. Soc., 1970, N 150, p. 35 - 44.

Kislyuk, V. S., Relke, H. 1992KFNT....8b..56K

Kolchinskii I.G., Onegina A.B.: 1977, Astrometry and Astrophysics, N33, 11 (in Ukraine).

Muminov M.M., Kahharov B.B., Yuldoshev K.H. et al.: 2013, Izvestija GA Pulkovo, 220, 517.

Pakuliak L.K., Andruk V.M., Golovnia V.V. et al.: 2016, Odessa Astron. Publ., 29, N1, 132.

Protsyuk Yu.I., Andruk V.N., Kazantseva L.V.: 2014, Odessa Astron. Publ., 27, N1, 59.

Protsyuk Yu.I., Andruk V.N., Kazantseva L.V. et al.: 2014, Odessa Astron. Publ., 27, N1, P..61.

Protsyuk Yu.I., Kovylianska O.E., Protsyuk S.V. et al.: 2014, Odessa Astron. Publ., 27, N1, 63.

Relke E., Protsyuk Yu.I., Andruk V.M. et al. 2015, Odessa Astron. Publ., 28, N2, 211.

Relke, H., Gamburg, O. Bulletin of the Institute of Astrophysics of the Academy of Science of Tadjikistan, N. 81, 1993.

Relke, H., Gamburg, O. Bulletin of the Institute of Astrophysics of the Academy of Science of Tadjikistan, N. 81, 1993.

Relke, H. Bulletin of the Institute of Astrophysics of the Academy of Science of Tadjikistan, N. 81, 1993.

Relke, H. 1991ATsir1551...36R

Yuldoshev Q.X., Ehgamberdiev Sh.A., Muminov M.M. et al.: 2017, Kinem. Phys. Cel. Bodies, 33, N5, 250. 